
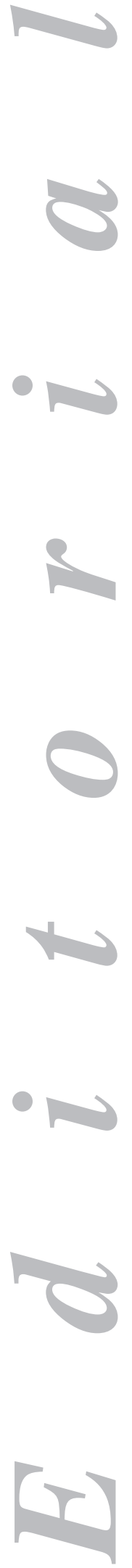

\section{Predicción de fibrosis hepática con pruebas no invasivas. Una apuesta de futuro}

Tanto el pronóstico como la posibilidad de tratamiento de las hepatopatías crónicas dependen, en gran medida, de la progresión de la fibrosis hepática que constituye la consecuencia final del daño crónico al hígado. Esta situación es dinámica y en ella se enfrentan dos procesos extremos: el de fibrogénesis y el de fibrolisis (1). Esto conlleva una acumulación de colágeno en el tejido, así como de otras proteínas de la matriz extracelular. El depósito progresivo de estas sustancias provoca finalmente la alteración de la morfología hepática, deteriora el funcionamiento del parénquima y acaba por inducir el desarrollo de hipertensión portal y sus secuelas.

En los últimos años se ha avanzado en el conocimiento de los mecanismos celulares y moleculares que llevan a la fibrogénesis, especialmente en lo referente a la importancia de los mediadores de la inflamación, la apoptosis o el papel de las células estrelladas (2). Asimismo, los mecanismos a través de los cuales la resistencia a la insulina induce el desarrollo de esteatosis y la consecuente aparición de fibrosis son, cada vez, mejor conocidos (3). Estos estudios han llevado a querer trasladar esta evidencia al campo de la clínica y al consiguiente desarrollo de marcadores biológicos que permitan una adecuada estadificación de la hepatopatía.

El modelo de desarrollo de fibrosis, sin embargo, necesita mejorar. La gran mayoría de los estudios de seguimiento utilizan modelos longitudinales, en los cuales se asume que la progresión de la fibrosis es un proceso lineal (4). Sin embargo, es muy probable que durante largos periodos de tiempo la situación permanezca estable y se produzcan reactivaciones temporales en las que el aumento de la actividad inflamatoria provocaría la progresión de la fibrosis (5).

La gran mayoría del conocimiento acerca del desarrollo de marcadores no invasivos de fibrosis hepática procede, por otra parte, de estudios transversales, de prueba diagnóstica. Para todos ellos, la prueba de referencia la constituye la biopsia hepática, una foto fija, en el contexto de un proceso largo y, muy probablemente, no lineal.

En los últimos 50 años, el empleo de la biopsia hepática se ha generalizado y ha permitido obtener un mejor conocimiento de las bases anatomopatológicas de las enfermedades hepáticas y de su evolución, y basar nuestros diagnósticos sobre datos objetivos. Sin embargo, este procedimiento no está exento de efectos deletéreos. La estancia hospitalaria se prolonga hasta en el 1 al 5\% de los pacientes sometidos a una BHP debido, principalmente, a complicaciones hemorrágicas. La mortalidad de la técnica se estima que es de 1/1.000 a 1/10.000 (6,7). Además, el riesgo de complicaciones es proporcional al número de pases de la aguja así como en presencia de determinadas condiciones del paciente, como en caso de sepsis o de los trastornos de la coagulación (8).

Las mayores limitaciones de la biopsia hepática son el error muestral y la variabilidad interobservador. En un adulto de complexión normal, la masa del teji- 
do hepático es aproximadamente de $1.500 \mathrm{~g}$. Una muestra de BHP representa una fracción del parénquima de tan sólo el 1/25.000 a 1/50.000, y el tamaño del cilindro hepático obtenido mantiene una relación inversamente proporcional con el error muestral. En los últimos años se han publicado varios estudios que demuestran que la fiabilidad del diagnóstico histológico depende del tamaño de la muestra analizada (9-11).

A los problemas de representatividad comentados se suman los derivados de la subjetividad de la valoración. Diversos estudios han mostrado que existe una gran variabilidad en la interpretación de las lesiones entre los diferentes patólogos (interobservador) e incluso en un mismo patólogo cuando valora una misma muestra en dos momentos diferentes (intraobservador). En el estudio METAVIR, 10 patólogos expertos en patología hepática examinaron 30 muestras de BHP de pacientes con hepatopatía por virus hepatitis $\mathrm{C}$ (VHC) y, aunque algunas lesiones fueron valoradas por todos ellos de forma superponible, en otras hubo marcadas diferencias. Entre estas últimas figuraban las necrosis erosivas, la actividad, las necrosis lobulillares y el índice de Knodell (12).

Por otra parte, en no pocas ocasiones, se desearía conocer la progresión de la enfermedad hepática a fin de valorar la respuesta al tratamiento. La limitación que supone repetir la biopsia hepática en términos de riesgo potencial para el paciente hace necesario el desarrollo de nuevas técnicas con las que evaluar la fibrosis hepática.

Por todos estos motivos, en los últimos años se han publicado numerosos estudios con diferentes marcadores biológicos, determinados en muestras de sangre o suero, que permiten la estadificación del grado de fibrosis sin necesidad de realizar una biopsia hepática.

En condiciones ideales, un marcador sérico de fibrosis debería ser específico de órgano, fácil de realizar, reproducible y barato. Además no debería servir únicamente para estadificar el grado de fibrosis sino también para monitorizar la progresión de la enfermedad y la eficacia del tratamiento.

Los marcadores séricos utilizados para el diagnóstico no invasivo de la fibrosis hepática pertenecen fundamentalmente a dos categorías: a) indirectos: son aquellos que no reflejan directamente el metabolismo de la matriz extracelular. En la mayoría de las ocasiones, se pretende además utilizar determinaciones que se realizan de manera rutinaria en estos pacientes con hepatopatías como los valores de transaminasas, el colesterol o el recuento plaquetario; y b) directos: generalmente constituidos por productos de degradación o síntesis de la matriz extracelular, como en el caso del péptido amino terminal del procolágeno III (PIIINP), la laminina, el ácido hialurónico, los inhibidores tisulares de las metaloproteasas (TIMP), etc.

Una de las principales limitaciones de los marcadores biológicos de fibrosis hepática es que se trata de determinaciones cuantitativas continuas que no necesariamente reflejan la complejidad del proceso fibrótico. Esto provoca, entre otras situaciones, que todos ellos permitan discriminar sin dificultad, la presencia de estadios extremos (F0 y F4) y que, sin embargo, en los niveles intermedios, exista un gran solapamiento de resultados que dificultan la correcta discriminación entre unos estadios y otros.

La gran mayoría de los estudios realizados cuyo objetivo es predecir el estadio de fibrosis hepática utilizando pruebas no invasivas, se han llevado a cabo en pacientes con hepatitis crónica C. En múltiples ocasiones se trata de marcadores séricos que combinan diferentes determinaciones analíticas. Así, disponemos en la actualidad de modelos como el APRI (13), el índice de Forns (14), el Fibrotest (15) o el modelo de Lok (16), por mencionar sólo algunos de los más conocidos. Este am- 
plio abanico de posibilidades lleva a concluir que, muy probablemente, ninguno de ellos por separado posee la suficiente validez diagnóstica para permitirnos predecir el estadio de fibrosis de forma suficientemente fiable. Así lo indica un metaanálisis publicado recientemente (17).

Por otra parte, se han desarrollado en los últimos tiempos, procedimientos basados en el análisis de la alteración de la elasticidad que la fibrosis produce en el parénquima hepático. Y así, asistimos a la aparición tanto de la elastografía transitoria (FibroScan, Echosens, París) como de la elastografía por resonancia magnética (ERM).

En el artículo publicado este mes en la Revista Española de Enfermedades Digestivas, Castiella y cols. (18) utilizan, en una muestra de pacientes con hemocromatosis fenotípica, el producto de la edad por la concentración de hierro hepático o índice de fibrosis y los valores de plaquetas, ferritina y transaminasas para analizar cuál de estos métodos es el mejor predictor del estadio de fibrosis hepática avanzada en esta población. La concentración de hierro hepático se evaluó bien en la muestra de biopsia, bien realizando una RM utilizando un protocolo específico. De entre los 32 pacientes analizados, en 22 (69\%) existía alguna mutación relacionada con la presencia de hemocromatosis hereditaria. La distribución por estadios de fibrosis fue: F0-2: 72\% y fibrosis avanzada (F3-4): $28 \%$. En 7 de los casos (22\%) se realizó determinación de la concentración de hierro hepático por RM $\mathrm{y}$, en todos los sujetos de la muestra se recogieron diferentes parámetros clínico-analíticos.

Los autores concluyen que el índice de fibrosis medido por biopsia hepática y el recuento plaquetario permiten descartar la presencia de fibrosis avanzada ( $\mathrm{F} \geq 3$ ) con elevada probabilidad (VPN: 94\%). Además, este valor aumenta hasta el 100\% cuando se combinan los resultados tanto del índice de fibrosis como del recuento plaquetario, con la cifra de transaminasas. El índice de fibrosis y el recuento plaquetario mostraron idéntica sensibilidad (86\%) mientras que la especificidad para cada una de las variables fue del 68 y $78 \%$, respectivamente.

Las principales aportaciones del mencionado estudio incluyen: a) el análisis de cuatro métodos diferentes para predecir el estadio de fibrosis hepática (recuento plaquetario, elevación de AST, ferritina sérica e índice de fibrosis) y la comparación de todos ellos con la prueba de referencia; b) la confirmación de la importancia de la exposición hepática al hierro en el desarrollo de fibrosis, como refleja el producto de la concentración de hierro hepático por la edad; y c) aumentar el conocimiento en lo referente al diagnóstico no invasivo de fibrosis hepática en los pacientes con hemocromatosis. Entre las potenciales limitaciones del estudio se encuentran: a) la recogida retrospectiva de los datos; b) la ausencia de información acerca de la existencia o no del enmascaramiento de los resultados de la biopsia hepática en el momento de realizar el estudio por RM; c) la información no publicada acerca de las características de las muestras de biopsia hepática, fundamentalmente longitud del cilindro y número de espacios porta, que inciden de manera determinante en el valor real de la estadificación de la fibrosis llevada a cabo por el patólogo; d) la necesidad de recurrir a la biopsia hepática, en parte de los pacientes de la muestra analizada, para realizar el cálculo de la concentración de hierro hepático; e) el escaso porcentaje de pacientes en los que se analiza el índice de fibrosis por RM, que genera amplios intervalos de confianza en los parámetros de validez de la prueba; f) un predominio de pacientes con estadios no avanzados de fibrosis $(\mathrm{F} \leq 2)$ que lleva a valores de la razón de verosimilitud positiva no lejanos de la unidad, traducido en un descenso en la sensibilidad de las pruebas (19); g) la no inclusión de un análisis de capacidad 
diagnóstica global de las pruebas no invasivas analizadas, habitualmente representada gráficamente en forma de curva ROC y, cuantitativamente, con el valor del área bajo dicha curva; y h) la ausencia de un análisis más complejo, multivariante, que justifique la combinación de pruebas de diagnóstico no invasivo.

Como ya se ha señalado, en los últimos tiempos se están llevando a cabo continuos esfuerzos para lograr diagnosticar el estadio de fibrosis hepática utilizando métodos que eviten al paciente la realización de una biopsia hepática.

En el caso concreto de la enfermedad hepática por depósito de hierro, se encuentra claramente establecido que dicha sobrecarga férrica posee un papel fundamental en el desarrollo de la fibrosis. De hecho, no es posible descartar completamente la presencia de fibrosis cuando las cifras de transaminasas son normales, habiéndose comunicado en pacientes con hemocromatosis hereditaria una prevalencia de fibrosis del $18 \%$ en este contexto (20).

La combinación de los valores de ferritina, el recuento plaquetario y la AST ha demostrado ser capaz de diagnosticar correctamente la presencia de cirrosis en el $81 \%$ de los casos de una muestra de pacientes homocigotos para la mutación C282Y (21). Previamente, otras variables clínico-analíticas sencillas, la ferritina, los niveles de AST y la hepatomegalia, habían demostrado ser útiles para descartar fibrosis avanzada (22). Asimismo, marcadores directos de fibrosis como el colágeno tipo IV sérico ha mostrado una buena correlación con el estadio de fibrosis hepática y una elevada sensibilidad para detectar fibrosis avanzada y cirrosis. Sin embargo, otros marcadores como la laminina y el TIMP-1, con cierta utilidad en otras hepatopatías, demostraron, en el mismo estudio, que poseían escasa sensibilidad (23).

En cuanto al papel de la RM en el estudio de la sobrecarga férrica, su utilidad parece demostrada tanto como para cuantificar el contenido de hierro hepático (24), como para generar un índice, constituido por el producto de la concentración de hierro hepático y la edad, con elevada sensibilidad y especificidad para predecir fibrosis avanzada (25). El grupo del propio Castiella, autor del artículo objeto de este editorial, corroboró estos datos en un análisis de validación externa (26).

Finalmente, la aparición de la elastografía transitoria (FibroScan ${ }^{\circledR}$ ) se ha utilizado para evaluar la fibrosis inducida por la sobrecarga férrica postransfusional, comunicándose una buena capacidad diagnóstica global para predecir fibrosis avanzada en este contexto, con un valor del área bajo la curva ROC de 0,82 (27). Además, recientemente, se ha publicado un estudio que compara los resultados de la elastografía transitoria y diversos marcadores séricos en sujetos homocigotos para C282Y frente a un grupo control. En ninguno de los sujetos de este último grupo, los valores del FibroScan sugerían la presencia de estadios de fibrosis $F \geq 2$. Aunque se observó correlación entre los valores de la elastografía y los marcadores séricos analizados, esta no se dio con los valores de ferritina sérica (28).

Los métodos no invasivos de predicción de fibrosis hepática poseen un futuro prometedor. Algunos de ellos, por su relativa accesibilidad y simplicidad, pueden llegar a convertirse en herramientas útiles para la práctica clínica diaria. Los métodos basados en técnicas de imagen parecen haber sido capaces de dar el salto cualitativo entre la letra impresa y la cabecera del paciente. Para los marcadores séricos esto se antoja más difícil, bien debido a la complejidad de las fórmulas generadas para su cálculo, bien porque alguna de las determinaciones que los componen pueden resultar costosas de obtener. En el caso concreto de los pacientes con sobrecarga férrica, la relativa baja prevalencia de la enfermedad supone dificultades añadidas, puesto que los reducidos tamaños muestrales que se obtienen limitan la 
generalización de los resultados y hacen necesarios estudios más amplios, probablemente multicéntricos.

En cualquier caso quizá no debamos considerar las pruebas no invasivas como sustitutos de la biopsia hepática. Ambas técnicas pueden complementarse y permitirnos una más completa evaluación del complejo proceso de la fibrosis en nuestros pacientes. El futuro nos podría llevar a realizar evaluaciones iniciales combinadas (biopsia y métodos no invasivos) para conocer la situación basal de la fibrosis y, posteriormente, en aquellos sujetos en los que se observe clara correlación, realizar el seguimiento únicamente con pruebas no invasivas.

\author{
J. C. Marín Gabriel y J. A. Solís Herruzo \\ Servicio de Medicina de Aparato Digestivo. Hospital Universitario 12 de Octubre. \\ Universidad Complutense de Madrid
}

\title{
BIBLIOGRAFÍA
}

1. Rockey DC, Bissell DM. Non-invasive measures of liver fibrosis. Hepatology 2006; 43 (2 Supl. 1): S113-S120.

2. Urtasun R, Nieto N. Hepatic stellate cells and oxidative stress. Rev Esp Enferm Dig 2007; 99 (4): 223-30.

3. Romero-Gómez M. Hepatitis C and insulin resistance: steatosis, fibrosis and non-response. Rev Esp Enferm Dig 2006; 98 (8): 605-15.

4. Poynard T, Bedossa P, Opolon P. Natural history of liver fibrosis progression in patients with chronic hepatitis C. Lancet 1997; 349 (9055): 825-32.

5. Hubscher SG. Histological grading and staging in chronic hepatitis: clinical applications and problems. J Hepatol 1998; 29 (6): 1015-22.

6. Froehlich F, Lamy O, Fried M, Gonvers JJ. Practice and complications of liver-biopsy - Results of a nationwide survey in Switzerland. Digestive Diseases and Sciences 1993; 38 (8): 1480-4.

7. Thampanitchawong P, Piratvisuth T. Liver biopsy: complications and risk factors. World J Gastroenterol 1999; 5 (4): 301-4.

8. Terjung B, Lemnitzer I, Dumoulin FL, Effenberger W, Brackmann HH, Sauerbruch T, et al. Bleeding complications after percutaneous liver biopsy. Digestion 2003; 67 (3): 138-45.

9. Colloredo G, Guido M, Sonzogni A, Leandro G. Impact of liver biopsy size on histological evaluation of chronic viral hepatitis: the smaller the sample, the milder the disease. J Hepatol 2003; 39 (2): 239-44.

10. Regev A, Berho M, Jeffers LJ, Milikowski C, Molina EG, Pyrsopoulos NT, et al. Sampling error and intraobserver variation in liver biopsy in patients with chronic HCV infection. Am J Gastroenterol 2002; 97 (10): 2614-8.

11. Bedossa P, Dargere D, Paradis V. Sampling variability of liver fibrosis in chronic hepatitis C. Hepatology $2003 ; 38$ (6): 1449-57.

12. Intraobserver and interobserver variations in liver biopsy interpretation in patients with chronic hepatitis $\mathrm{C}$. The French METAVIR Cooperative Study Group. Hepatology 1994; 20 (1 Pt 1): 15-20.

13. Wai CT, Greenson JK, Fontana RJ, Kalbfleisch JD, Marrero JA, Conjeevaram HS, et al. A simple noninvasive index can predict both significant fibrosis and cirrhosis in patients with chronic hepatitis C. Hepatology 2003; 38 (2): 518-26.

14. Forns X, Ampurdanes S, Llovet JM, Aponte J, Quinto L, Martinez-Bauer E, et al. Identification of chronic hepatitis C patients without hepatic fibrosis by a simple predictive model. Hepatology 2002; 36 (4): 986-92.

15. Imbert-Bismut F, Ratziu V, Pieroni L, Charlotte F, Benhamou Y, Poynard T, et al. Biochemical markers of liver fibrosis in patients with hepatitis $C$ virus infection: a prospective study. Lancet 2001; 357 (9262): 1069-75.

16. Lok ASF, Ghany MG, Goodman ZD, Wright EC, Everson GT, Sterling RK, et al. Predicting cirrhosis in patients with hepatitis C based on standard laboratory tests: Results of the HALT-C cohort. Hepatology 2005; 42 (2): 282-92.

17. Parkes J, Guha IN, Roderick P, Rosenberg W. Performance of serum marker panels for liver fibrosis in chronic hepatitis C. J Hepatol 2006; 44 (3):462-74.

18. Castiella A, Zapata E, Otazua P, Fernández J, Alustiza JM, Ugarte M, et al. Utilidad de los diferentes métodos no invasivos de predicción de fibrosis hepática en pacientes del País Vasco con hemocromatosis fenotípica. Rev Esp Enferm Dig 2008; 100 (10): 611-4.

19. Jaeschke R, Guyatt GH, Sackett DL. Users' guides to the medical literature. III. How to use an article about a diagnostic test. B. What are the results and will they help me in caring for my patients? The Evidence-Based Medicine Working Group. JAMA 1994; 271 (9): 703-7. 
20. Beaton M, Adams PC. Assessment of silent liver fibrosis in hemochromatosis $\mathrm{C} 282 \mathrm{Y}$ homozygotes with normal transaminase levels. Clin Gastroenterol Hepatol 2008; 6 (6): 713-4.

21. Beaton M, Guyader D, Deugnier Y, Moirand R, Chakrabarti S, Adams P. Noninvasive prediction of cirrhosis in C282Y-linked hemochromatosis. Hepatology 2002; 36 (3): 673-8.

22. Guyader D, Jacquelinet C, Moirand R, Turlin B, Mendler MH, Chaperon J, et al. Noninvasive prediction of fibrosis in C282Y homozygous hemochromatosis. Gastroenterology 1998; 115 (4): 929-36.

23. George DK, Ramm GA, Walker NI, Powell LW, Crawford DH. Elevated serum type IV collagen: a sensitive indicator of the presence of cirrhosis in haemochromatosis. J Hepatol 1999; 31 (1): 47-52.

24. Alústiza JM, Artetxe J, Castiella A, Agirre C, Emparanza JI, Otazua P, et al. MR quantification of hepatic iron concentration. Radiology 2004; 230 (2): 479-84.

25. Olynyk JK, St Pierre TG, Britton RS, Brunt EM, Bacon BR. Duration of hepatic iron exposure increases the risk of significant fibrosis in hereditary hemochromatosis: a new role for magnetic resonance imaging. Am J Gastroenterol 2005; 100 (4): 837-41.

26. Castiella A, Emparanza JI. External validation for fibrosis predicting index in hereditary hemochromatosis. Am J Gastroenterol 2005; 100 (10): 2366-7.

27. Mirault T, Lucidarme D, Turlin B, Vandevenne P, Gosset P, Ernst O, et al. Non-invasive assessment of liver fibrosis by transient elastography in post transfusional iron overload. Eur J Haematol 2008; 80 (4): 337-40.

28. Adhoute X, Foucher J, Laharie D, Terrebonne E, Vergniol J, Castéra L, et al. Diagnosis of liver fibrosis using FibroScan and other noninvasive methods in patients with hemochromatosis: a prospective study. Gastroenterol Clin Biol 2008; 32 (2): 180-7. 